\title{
Effects of hypoxia on rat mesenchymal stem cells
}

\author{
Xiaoli $\mathrm{Li}^{1}$, Lei Yang ${ }^{1}$, Tianqing Liu ${ }^{1}$, Xuehu Ma ${ }^{1}$, Zhanfeng Cui ${ }^{1}$ \\ ${ }^{l}$ Dalian R\&D Center for Stem Cell and Tissue Engineering, Dalian University Of Technology, China
}

Rat mesenchymal Stem Cells (MSCs) represent a small portion of the cells in the stromal compartment of bone marrow, capable of differentiating in vitro into mature-like cells from all three germ layers. They have the potential to differentiate into cartilage cells, cardiac muscle cells, adipose cells, nerve cells and osteoblasts. On the other hand, the physiologic oxygen tension inside the body is much lower and varies from tissue to tissue, ranging from $1 \%$ in cartilage and $1 \%$ to $7 \%$ in BM to a maximum of $10-13 \%$ in the arteries, lungs, and liver. To mimic the in vivo conditions, it is necessary to examine the role of the oxygen tension in regulating the capacity of MSCs both to selfrenew and to maintain their pluripotentiality (stemness).

SD rat mesenchymal stem cells were isolated with density gradient centrifugation. rMSCs were grown under low$\mathrm{pO}_{2}$ conditions (5 and $10 \%$ oxygen) or air $\left(21 \%\right.$ oxygen, $\left.5 \% \mathrm{CO}_{2}\right)$. The cell growth curves were obtained and colony counts were carried out to examine the role of different $\mathrm{pO}_{2}$ on $\mathrm{rMSC}$ proliferation. Then the expanded cells were induced to osteoblasts, cartilage cells and adipocytes to confirm their multi-differentiate properties by ALP, Von Kossa, Oil Red-O and Toluidine blue staining. The results showed that oxygen tension affected cell proliferation both in primary culture and in subculture. At all $\mathrm{pO}_{2}$ levels lower than that of air, we observed a significant increase in cell number. Moreover, MSCs grown at $5 \% \mathrm{O}_{2}$ consistently showed a nearly two-fold increase compared to those at other pO2. And their proliferation potential and metabolic activity were higher for cultures maintained in low oxygen than those in control oxygen. These findings demonstrate that hypoxia can promote the proliferation of rMSCs in vitro. MSCs maintained the ability to thrive in hypoxic conditions suggesting that hypoxia may be an essential element of the in vivo MSCs niche.

Keywords: hypoxia, mesenchymal stem cells, proliferation, differentiation, HIF-1 $\alpha$

Cell Research (2008) 18:s170. doi: 10.1038/cr.2008.260; published online 4 August 2008

Correspondence: Xiaoli Li

E-mail: ningmengguo2001@yahoo.com.cn 